\title{
HOW DOES ALGORITHM VISUALIZATION AFFECT COLLABORATION? Video Analysis of Engagement and Discussions
}

\author{
Ari Korhonen \\ Department of Computer Science and Engineering, Helsinki University of Technology \\ P.O. Box 5400, FI-02015 TKK, Finland \\ archie@cs.hut.fi \\ Mikko-Jussi Laakso \\ Department of Information Technology, University of Turku, FI-22014 Turun Yliopisto, Finland \\ milaakso@utu.fi \\ Niko Myller \\ Department of Computer Science and Statistics, University of Joensuu, P.O. Box 111, FI-80101 Joensuu, Finland \\ niko.myller@cs.joensuu.fi
}

\begin{abstract}
Keywords: Extended engagement taxonomy, Collaborative learning, Algorithm animation, Visual algorithm simulation.
Abstract: In this paper, we report a study on the use of Algorithm Visualizations (AV) in collaborative learning. Our previous results have confirmed the hypothesis that students' higher engagement has a positive effect on learning outcomes. Thus, we now analyze the students' collaborative learning process in order to find phenomena that explain the learning improvements. Based on the study of the recorded screens and audio during the learning, we show that the amount of collaboration and discussion increases during the learning sessions when the level of engagement increases. Furthermore, the groups that used visualizations on higher level of engagement, discussed the learned topic on different levels of abstraction whereas groups that used visualizations on lower levels of engagement tended to concentrate more on only one aspect of the topic. Therefore, we conclude that the level of engagement predicts, not only the learning performance, but also the amount of on-topic discussion in collaboration. Furthermore, we claim that the amount and quality of discussions explain the learning performance differences when students use visualizations in collaboration on different levels of engagement.
\end{abstract}

\section{INTRODUCTION}

Empirical evaluations have yielded mixed results when determining the usefulness of Algorithm Visualizations (AV) with empirical experiments. The meta-analysis by (Hundhausen et al., 2002) concluded that the activities performed by the students are more important than the content of the visualization. This has led to the proposition of Engagement Taxonomy by (Naps et al., 2002) to characterize the different levels of activities the students can perform with AV. The taxonomy is based on the Cognitive Constructivist learning theory (Hundhausen et al., 2002; Garrison, 1993; Piaget, 1977) and a student is supposed to achieve better learning results on higher engagement levels. Moreover, (Myller et al., 2008) have developed the taxonomy further by introducing
Extended Engagement Taxonomy (EET), which describes the levels of engagement in finer level of detail. Furthermore, they have correlated the qualities of students' collaboration processes to different EETlevels, and therefore, extended the taxonomy into the direction of Social Constructivism (Palincsar, 1998; McMahon, 1997; Vygotsky, 1978).

Collaborative learning has become popular in Computer Science education (Beck and Chizhik, 2008; Teague and Roe, 2008; Valdivia and Nussbaum, 2007). Although visualizations have been employed in collaborative learning, collaboration introduces new challenges for the visualization tools. For example, the exchange of experiences and ideas, and coordination of the joint work are needed when students are no longer working individually (Suthers and Hundhausen, 2003). Furthermore, visualizations can 
provide a shared external memory that can initiate negotiations of meanings and act as a reference point when ideas are explained or misunderstandings are resolved (Suthers and Hundhausen, 2003). This implies that also new theories or extension of the previous ones are needed to guide the development and research of the visualization tools for collaborative learning.

In this paper, we study the use of AV in collaborative learning. We have utilized EET as a framework to test the impact of engagement levels on the learning process when the students work in pairs. In this experimental study, students collaborating on different engagement levels were compared with each other while they were learning concepts related to binary heaps. This is a follow-up study in a series of studies. The previous studies have shown that the engagement levels have a role to play in learning and showed that the use of visualizations on higher levels of engagement improves learning results (Laakso et al., 2009; Myller et al., 2007). However, this further investigation revealed new results that support the view that higher engagement levels have an effect not only on learning outcomes, but also on the amount of collaboration or discussion students have during the learning sessions. In other words, the engagement seems to have an effect not only on the student-content interaction, but also on the student-student interaction (see (Moore, 1989)). We hypothesize that these two together have influenced the students learning results.

Although a plethora of studies that concentrate on students' performance (Grissom et al., 2003; Naps and Grissom, 2002; Naps et al., 2002; Hundhausen et al., 2002) exist, we also need to understand the learning process and how the visualizations affect it (Hundhausen, 2002; Hundhausen and Brown, 2008). This information is essential when developing new systems in order to enhance students' learning with algorithm visualizations.

The structure of this paper is as follows: section 2 presents previous work on visualizations, engagement and interaction. The setup and design of the study are described in section 3 . In section 4 , the results are presented and they are further discussed in section 5 . Finally, conclusions and future directions are given in section 6 .

\section{PREVIOUS WORK}

\subsection{Engagement}

As an attempt to describe the mixed results of previous research in AV usage (Hundhausen et al., 2002) in learning and teaching of algorithms and data structures, Engagement Taxonomy (ET) was introduced by (Naps et al., 2002). The central idea of the taxonomy is that the higher the engagement between the learner and the visualization, the higher the positive effects on learning outcomes. ET consists of six levels of engagement between the user and the visualization:

No viewing - There is no visualization to be viewed.

Viewing - The visualization is only looked at without any interaction.

Responding - Visualization is accompanied with questions, which are related to the content of the visualization.

Changing - Modification of the visualization is allowed, for example, by varying the input data set or algorithm simulation.

Constructing - Visualization of program or algorithm is created.

Presenting - Visualizations are presented to others for feedback and discussion.

ET has been used in the development of AV tools and several studies have utilized the framework and provided further support for it (?, see, e.g.,)]Grissom2003, Grissom2002. There are also other studies which have shown that visualizations improve learning results, without actually utilizing the ET framework in the design of the study (BenBassat Levy et al., 2003). In addition to this, research in educational psychology and multimedia learning have received similar results (Evans and Gibbons, 2007).

Although there is some anecdotal evidence on how the visualizations could affect collaborative learning process (Hundhausen, 2002; Hundhausen, 2005 ), there have been very few formal studies investigating it, especially from the point of view of engagement (Hundhausen and Brown, 2008). In this paper, we aim to research how the engagement between the learners and the visualization affects the interactions (i.e. collaboration and discussion) between learners.

(Myller et al., 2008) have proposed an extension to the ET called Extended Engagement Taxonomy (EET). The idea of this extension is to let the designers and researchers of visualizations to use finer granularity of engagement levels in their tools and experimental designs. They provide the following engagement levels to be used together with the original ones: controlled viewing, providing input, modification, and reviewing. In this study, we will utilize the controlled viewing level in order to make a difference between 
the visualizations that can only be viewed by the student (EET level: viewing, e.g. static visualizations or animations with only a playing option) compared to those which can be controlled (EET level: controlled viewing, e.g., animations with VCR-like controls in order to step and play the animation both forwards and backwards).

\subsection{TRAKLA2}

TRAKLA2 is a practicing environment for visual algorithm simulation exercises (Korhonen et al., 2003; Malmi et al., 2004) that are automatically assessed tracing exercises solved by a student in a web-based learning environment. The system distributes individually tailored exercises to students and provides instant feedback on students' solutions. In visual algorithm simulation exercises, a student directly manipulates the visual representations of the underlying data structures. Thus, the student actually manipulates real data structures through operations of the graphical user interface (GUI) with the purpose of performing the same changes on the data structures as the actual algorithm would perform. Each change leads the data structure to a new state. An answer to an exercise is a sequence of these states, and the task is to perform the correct operations that will simulate the running of the algorithm.

Each TRAKLA2 exercise consists of a description of the exercise accompanied with pseudo-code representation of the algorithm, and possibly support material that introduces the theory and examples of the algorithm in question, instructions on how to interact with the GUI, and an interactive Java applet that is utilized to enter the answer. The current exercise set consists of over 50 assignments on basic data structures, search structures, hashing methods as well as sorting and graph algorithms.

Example: Let us consider the exercise in Figure 1. The student is supposed to manipulate the visual representation(s) of the Binary Heap data structure by invoking context-sensitive drag-and-drop operations. The idea is to simulate the linear time BuildHeap algorithm. The manipulation can be done in either of the representations shown in the figure (i.e. the array or the binary tree representation). A key can be shifted up in terms of swap operations with its parent until the heap property is satisfied (the key at each node is smaller than or equal to the keys of its children). A single swap operation is performed by dragging and dropping a key in the heap on top of another key

An exercise applet is initialized with randomized input data. The BuildHeap exercise, for example, is initialized with 15 numeric keys that correspond to the priority values. The student can reset the exercise by pressing the Reset button at any time. As a result, the exercise is reinitialized with new random keys. When attempting to solve the exercise, the student can review the answer step by step using the Animator panel. Moreover, the student can Submit the answer for immediate assessment and feedback. The feedback reports the number of correct steps out of the total number of steps in the exercise. This kind of automatic assessment is possible due to the fact that the student is manipulating real data structures through the GUI. Thus, it is possible to implement the same algorithm the student is simulating, and execute it so that the algorithm manipulates the same data structures with same data, but different instances, as the student. Therefore, the assessment is based on the comparison of the two instances of the same data structures with each other.

An exercise can be submitted an unlimited number of times. However, a solution for a single instance of an exercise with certain input data can be submitted only once. In order to resubmit a solution to the exercise, the student has to reset the exercise and start over with new randomized input data. A student can also review a Model answer for each attempt. It is represented in a separate window as an algorithm animation accompanied with a pseudo code animation so that the execution of the algorithm is visualized step by step. The states of the model solution can be browsed back and forth using a similar animator panel as in the exercise. For obvious reasons - after opening the model solution - the student cannot submit a solution until the exercise has been reset and resolved with new random data.

\subsection{Our Previous Studies on the Same Topic}

The study reported in this paper belongs to a series of studies that have been run since autumn 2006 (Laakso et al., 2009; Myller et al., 2007). This is actually a follow-up video analysis of an experiment that we carried out in spring 2007 (Laakso et al., 2009). The objective of the experiment was to compare the learning outcomes of students who collaboratively used algorithm visualizations on two different EET levels, namely controlled viewing and changing. The results in sections 2.3.1 and 2.3.2 have already been reported and explained in more detail elsewhere (Laakso et al., 2009) but are given here in order to allow the discussion of them in relation to the findings that are reported in this paper in section 4. In the sections 2.3.1 and 2.3.2, the analysis was done for all the partic- 

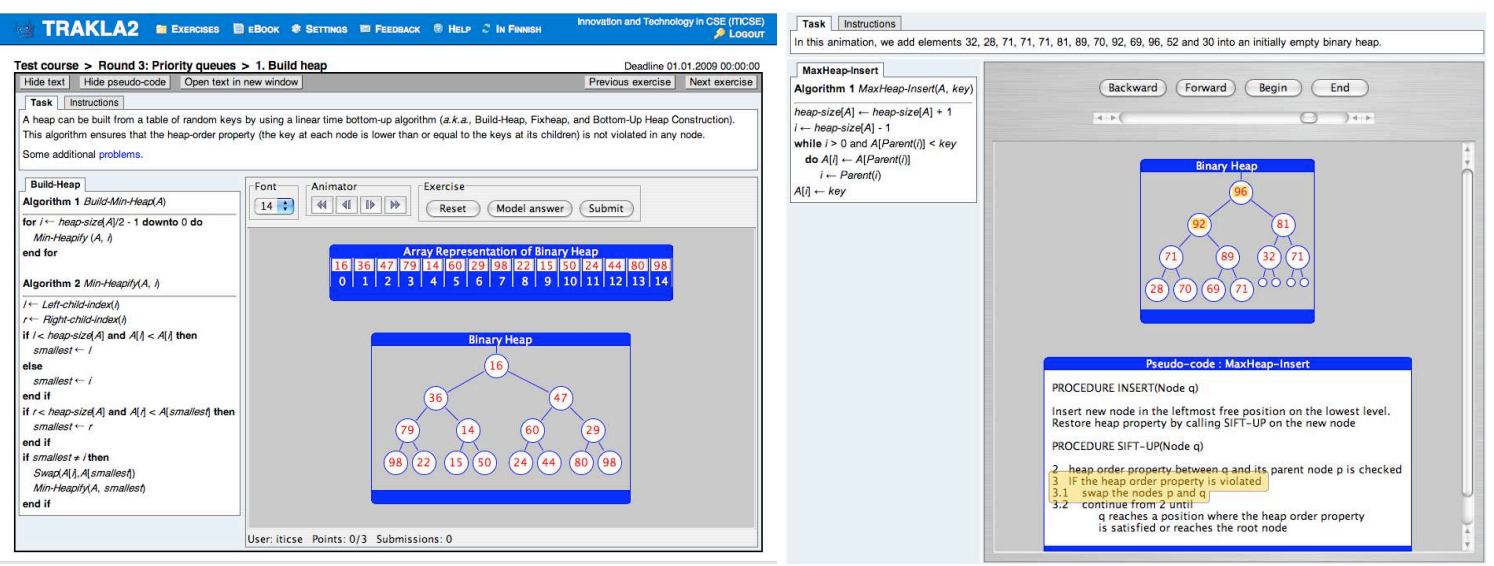

Figure 1: TRAKLA2 algoritm simulation exercise is on the left and corresponding model answer animation on the right.

ipants or groups of the same experiment that is reported in this paper. See section 3 for further description of the study design.

\subsubsection{Learning Results}

The pre- and post-test results for both conditions are given in Table 1. Based on the two-tailed t-test, the differences in the pre-test scores between conditions were not statistically significant meaning that the students' preliminary knowledge on the topic was similar. The differences in the post-test scores between conditions, both individual and group averages, were statistically significant based on the one-tailed t-test $(t(69)=-1.73, p<0.05$ and $t(31)=-1.97, p<$ 0.05 , respectively). We used the one-tailed t-test to analyze the post-test scores because of our hypothesis that the treatment group was expected to perform better. This hypothesis was formed based on the Engagement Taxonomy (Naps et al., 2002) and the previous results in similar studies performed by others (Grissom et al., 2003; Hundhausen and Brown, 2008; Naps and Grissom, 2002) and us (Myller et al., 2007).

Table 1: The pre- and post-test results between conditions (standard deviations are given in parentheses) $(n=71)$.

\begin{tabular}{lccc}
\hline & Pre-test & $\begin{array}{c}\text { Post-test } \\
\text { individual avg group avg }\end{array}$ & $\begin{array}{c}\text { Post-test } \\
\text { group av }\end{array}$ \\
\hline \hline Control & $8.9(6.1)$ & $30.5(6.5)$ & $30.4(4.6)$ \\
Treatment & $9.3(5.7)$ & $33.3(6.7)$ & $33.5(4.3)$ \\
\hline
\end{tabular}

\subsubsection{Time Allocation between Engagement Levels}

Table 2 presents the distribution of the average times spent on each EET level. This was measured by watching the videos and marking times when the EET level changed from one to another, and then summing up the times on each EET level. Based on this analysis, we made the final classification of groups into different conditions, because although some students were originally assigned to treatment condition, in which they were supposed to work on changing level, they never did, and therefore belonged to the control condition. This also shows that the amount of time that students spent on reading or looking at static images is almost the same in both groups and only the looking at the animations, which they could control, and the algorithm simulation exercises were used differently. In the control condition, the animations were the only active form of engagement whereas in treatment condition they also had the option of solving algorithm simulation exercises. The latter was more important due to the fact that this group used animations almost only for figuring out how they should simulate the algorithm.

Table 2: The distribution of time between EET levels (standard deviations are given in parentheses) $(n=35)$.

\begin{tabular}{lcc}
\hline & Control & Treatment \\
\hline \hline No viewing & $48.1 \%(15.8)$ & $43.2 \%(19.2)$ \\
Viewing & $38.3 \%(15.8)$ & $38.1 \%(13.1)$ \\
Controlled viewing & $13.8 \%(6.0)$ & $5.1 \%(6.0)$ \\
Changing & $0.0 \%(0.0)$ & $12.6 \%(2.0)$ \\
\hline
\end{tabular}

Table 3: The number of times each EET level is entered (standard deviations are given in parentheses) $(n=35)$.

\begin{tabular}{lcc}
\hline & Control & Treatment \\
\hline \hline No viewing & $6.9(2.1)$ & $6.2(1.7)$ \\
Viewing & $7.7(3.4)$ & $6.7(3.2)$ \\
Controlled viewing & $5.2(2.8)$ & $2.4(2.6)$ \\
Changing & $0.0(0.0)$ & $4.1(1.6)$ \\
\hline
\end{tabular}

Table 3 shows how many times students used ma- 
terials on each EET level. For example, students in the control group used user-controlled animations (controlled viewing) 5 times on average, whereas students in the treatment group used them 2 or 3 times on average. This also shows the shift from the use of animations to the algorithm simulations in the treatment condition.

\section{METHODOLOGY}

This is a follow-up analysis for the quantitative study (Laakso et al., 2009), in which we showed that the use of higher engagement levels has an positive effect on the students' learning outcomes. Thus, the description of the experiment in this section is in many ways similar to the previous report. However, as we analyze the learning process - not its outcomes - the methodology is naturally different.

The objective in this study is to compare the learning processes of students who collaboratively used algorithm visualizations on two different EET levels, namely controlled viewing and changing. This is an observational study based on screen capture and audio recording analysis of students' interactions during the experiment. Students' activities were recorded utilizing a screen capturing software. The recordings were accompanied by an audio track and thus, contained on-screen activities, i.e., mouse movements, keyboard typings, scrolling of the tutorial page back and forth in the browser window, as well as the conversation between the pair members.

\subsection{Participants}

Students were mainly first year students, however, some students from other years were also on the course. All students had previously been using TRAKLA2 during the course to complete three assignment rounds related to basic data structures (e.g. lists, stacks and queues) and algorithm analysis, sorting and binary tree traversing. Thus, all students should have known how to use TRAKLA2, been familiar with its visualizations and all its features that were needed to complete the assignments.

Students were randomized to the computer lab sessions and sessions were randomly assigned to each condition with the limitation that parallel sessions belonged to different EET levels. The total number of participating students was 92. However, not all of them allowed to monitor their performance, nor were they willing to do group work. In addition, in some of the workstations, the Java applet was not working properly and there were problems in data cap- ture. Thus, the total number of participants (students) was 71 , divided into 7 groups (sessions). The original number of lab sessions was 8 , but the last one (that would have been a control group) was excluded because it was an English speaking group, and the materials were mostly in Finnish.

The study was performed at the computer lab sessions that lasted for 2 hours, and they were run on two days in two consecutive weeks. Each day, there were two times two sessions with different conditions (control and treatment) running simultaneously. There were 10 to 15 participants in each session in both conditions. The external conditions, such as noise level, were similar in all sessions and based on the video and audio analysis it did not affect the learning process.

\subsection{Procedure}

In the beginning of the session, students took the individual pre-test, in which they answered questions related to binary heaps in 15 minutes. There were 9 simple questions about binary heaps, which could be answered with a few words, and one question asked students to draw a binary heap's tree representation. After this, they freely formed pairs with their peers and gave their consent to participate in the experiment and to be monitored during the experiment. If there was an odd number of students, one group consisted of 3 students. Each pair was allocated to a single computer.

After the pre-test, students had 45 minutes to go through the learning materials of their condition. The collaboration was monitored by recording their talking and capturing their activities on the computer screens. In addition, in this learning phase student were given three paper-and-pencil assignments. The session ended with an individual post-test. The students were given 30 minutes to answer the questions in the post-test. The post-test contained six questions which were the same as in the pre-test, and in addition to that, there were seven questions that were more difficult and comparable to the questions students needed to answer during the learning session.

Each question in the pre- and post-tests was analyzed on a scale from 0 and 4 . Zero points meant less than 25 percent of the answer was correct in the answer, and each point meant a 25 percent increase in the correctness of the answer.

\subsection{Method}

In this overt research method, we observed the students in their activities, i.e., by watching the recordings afterwards (Gall et al., 2006). Participants were 
Computer Science major students on a data structures and algorithms course at Helsinki University of Technology. The students worked in pairs, and they were aware of being observed. We asked a permission to monitor them in advance.

We utilized TRAKLA 2 in order to provide the students with algorithm simulation exercises that act on the EET level changing (i.e, treatment group). However, the students did not have the option to reset the exercise in order to obtain a new similar exercise with new input data, but they had to work with a fixed input data for each exercise for the whole session. The animations that the students used on controlled viewing level (i.e., control group) were similar to the model answers provided by the TRAKLA2 system.

There was a total of 35 videos (about 45 minutes each), and we included three videos from both conditions into this analysis, in total six videos. From each video, we randomly selected a clip about $20 \mathrm{~min}-$ utes long that contained activities on all applicable engagement levels. The videos were analyzed in five second time slots that were classified according to the following four factors.

The first factor classified the engagement level according to the extended engagement taxonomy: no viewing (e.g., reading phase), viewing (e.g., watching figures), controlled viewing (e.g., watching animations or model solutions step-by-step with user in control), and changing (i.e., solving an algorithm simulation exercise). However, if the students were solving the paper-and-pencil exercises, these episodes were classified into a separate class that was not used in the analysis. The second factor categorized each time slot based on audio analysis and determined whether the pair was having a conversation (or if they were silent). The third aspect specified the content of the conversation according to the following six categories: algorithm and data structure (DS) behavior (e.g., students discuss the features of binary heap), the tool and its features (e.g., students discuss how to use the tool), exercise (e.g., students discuss how to solve the exercise), referring to the learning materials (e.g., students are reading parts of the learning material out loud and then discussing that part of the materials), on-topic (i.e., students are discussing something that is related to the learning but does not belong to any other category) and off-topic (i.e., student are discussing something that does not relate to the learning process in any way).

Three different persons classified randomly selected videos with the restriction that each person analyzed at least one video from the control group and one from the treatment group.

\section{RESULTS}

In this section, we present the results of our study in which we analyzed the students' behavior during their learning process. Six groups were randomly selected (three groups from both conditions) from a total of 35 groups. We analyzed a 20-minute-long clip of screen capturing video and audio for each group in order to collect the amount of discussions, their contents, and the EET-level at each moment in order to understand the differences in the amount of discussions and their contents between the engagement levels.

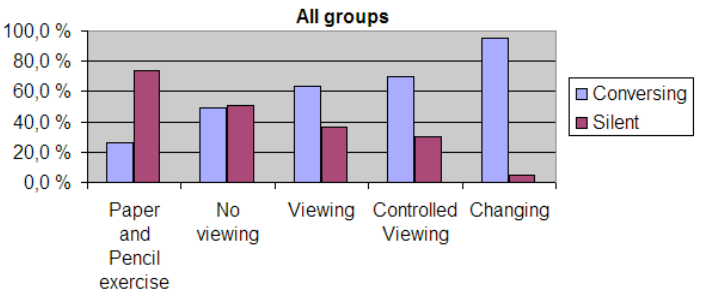

Figure 2: Distribution of activities in all groups on all EETlevels.

Figures 2, 3 and 4 show the distributions between the percentages of time that the students were conversing and silent. Based on the figures, one can see that the amount of conversation increases when the engagement level increases. This was also confirmed by using the $\chi^{2}$-test on counts (all: $\chi^{2}(4)=$ $330.5, p<.001$, control: $\chi^{2}(4)=84.1, p<.001$, treatment: $\left.\chi^{2}(4)=134.4, p<.001\right)$, which showed that the engagement level has an effect on the amount of discussion, overall and in each condition.

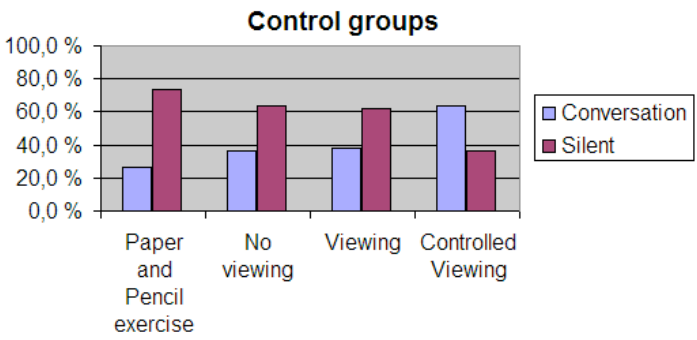

Figure 3: Distribution of activities for control groups on all EET-levels.

Pairwise comparison of the distributions on each EET level between conditions (see Figure 5) with the $\chi^{2}$-test on counts showed that the distributions were different on levels no viewing $\left(\chi^{2}(1)=9.2, p<.001\right)$, viewing $\left(\chi^{2}(1)=24.4, p<.001\right)$ and controlled viewing $\left(\chi^{2}(1)=21.4, p<.001\right)$, but not when students were doing a paper and pencil exercise. The test could not be performed on the changing level as it was only available to treatment condition. 
Table 4: Discussion content for all groups on all EET-levels.

\begin{tabular}{lcccccccc}
\hline & $\begin{array}{c}\text { Alg. \& DS } \\
\text { behavior }\end{array}$ & $\begin{array}{c}\text { Code } \\
\text { reading }\end{array}$ & $\begin{array}{c}\text { Exercise } \\
\text { beheferring to } \\
\text { learning mat. }\end{array}$ & Tool Coordination & $\begin{array}{c}\text { On } \\
\text { Off } \\
\text { topic } \\
\text { topic }\end{array}$ \\
\hline \hline $\begin{array}{l}\text { Paper and pencil } \\
\text { exercise }\end{array}$ & $65.2 \%$ & $0.9 \%$ & $11.3 \%$ & $0.0 \%$ & $0.0 \%$ & $15.7 \%$ & $1.7 \%$ & $5.2 \%$ \\
\hline $\begin{array}{l}\text { No viewing } \\
\text { Viewing }\end{array}$ & $39.2 \%$ & $14.9 \%$ & $5.4 \%$ & $14.2 \%$ & $4.1 \%$ & $6.8 \%$ & $10.1 \% 5.4 \%$ \\
Controlled & $32.8 \%$ & $24.8 \%$ & $5.1 \%$ & $4.4 \%$ & $2.2 \%$ & $7.3 \%$ & $16.8 \%$ & $6.6 \%$ \\
viewing & $68.7 \%$ & $19.4 \%$ & $0.9 \%$ & $3.3 \%$ & $1.9 \%$ & $3.8 \%$ & $0.0 \%$ & $1.9 \%$ \\
Changing & $65.0 \%$ & $0.0 \%$ & $7.8 \%$ & $0.0 \%$ & $13.8 \%$ & $1.8 \%$ & $9.7 \%$ & $1.8 \%$ \\
\hline
\end{tabular}

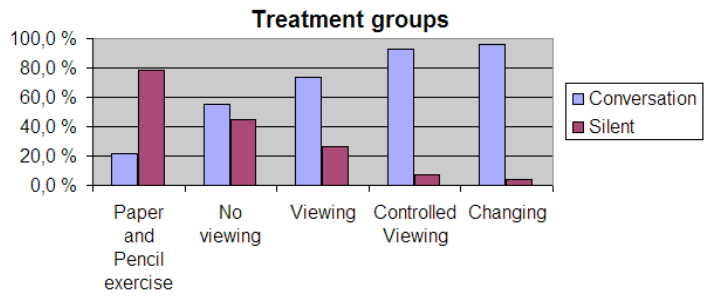

Figure 4: Distribution of activities for treatment groups on all EET-levels.

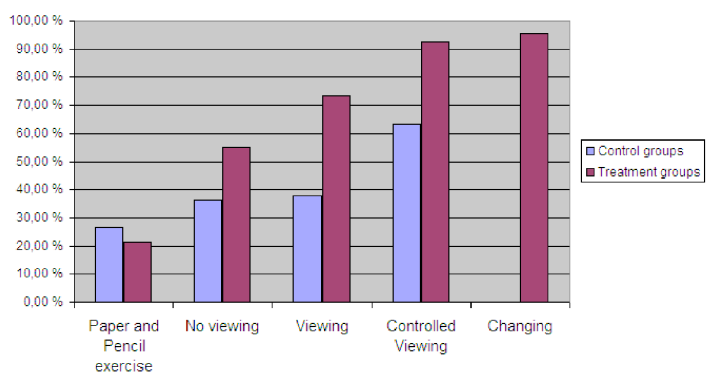

Figure 5: The percentages of time that students were discussing on each EET-level. The rest of the time students were silent. The control group does not have a value for the changing level, because it was unavailable for them.

Tables 4 and 5 show the distributions of the discussion contents on each engagement level and in each condition. When looking at the overall distribution, one can observe that the distributions of the discussion contents are similar on the controlled viewing and changing levels and when students are doing the paper and pencil exercises. Similarly, the distributions of no viewing and viewing seem more alike.

However, when the distributions between the conditions are compared, it can be seen that the no viewing, viewing and controlled viewing levels induce different kinds of discussions between the conditions. In control condition, the discussions are more related to the algorithm and data structure (DS) behavior, whereas in treatment condition larger proportions of the discussions on these levels are related to the pseudo code reading. In treatment condition, the changing level seems to be similar to the controlled viewing level and the paper and pencil exercise doing.

\section{DISCUSSION}

In this study, we have investigated the collaboration process when students were learning with visualization on different engagement levels. We can conclude that higher engagement with the visualization has a positive effect on students interaction with each other. Moreover, it seems that when students work on a larger number of engagement levels, their collaboration and communication is further improved.

Our results support the findings of (Hundhausen and Brown, 2008; Hundhausen, 2002), i.e., the higher engagement level between the visualization and learners increases the peer-to-peer (or student-student by (Moore, 1989)) communication. Students are more actively involved as the engagement level increases. Based on the results, we can say that if students work on higher engagement levels, their activities also positively change on lower levels. This phenomenon can be easily observed when we investigate the changes in the amount of discussion in the Figures 3 and 4 on controlled viewing and viewing between control and treatment groups. When students were working on changing level in the treatment group, the amount of silence dramatically decreased as the engagement level increased. At the two highest levels, the silence is practically absent. In control condition, the amount of silence decreases, but the change is smaller. For example, there is over $30 \%$ of the time when students are silent on controlled viewing level in control group while the time of being silent is well below $10 \%$ for the treatment group. The same difference is much more drastic in the viewing-level between the groups. Our understanding is that this is due to the fact that while students are solving a paper-and-pencil or TRAKLA2 algorithm simulation exercise, they realize that they cannot solve it. Therefore, they need to go back to the learning materials or the correspond- 
Table 5: Discussion content for control and treatment groups on all EET-levels.

\begin{tabular}{|c|c|c|c|c|c|c|c|c|}
\hline & $\begin{array}{c}\text { Alg. \& DS } \\
\text { behavior }\end{array}$ & $\begin{array}{c}\text { Code } \\
\text { reading }\end{array}$ & Exercise & $\begin{array}{l}\text { Referring to } \\
\text { learning mat }\end{array}$ & Tool & Coordination & $\begin{array}{c}\text { On } \\
\text { topic }\end{array}$ & $\begin{array}{c}\text { Off } \\
\text { topic }\end{array}$ \\
\hline \multicolumn{9}{|l|}{ "CONTROL } \\
\hline $\begin{array}{l}\text { Paper and pencil } \\
\text { exercise }\end{array}$ & 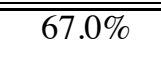 & $0.9 \%$ & $11.6 \%$ & $0.0 \%$ & $0.0 \%$ & $13.4 \%$ & $1.8 \%$ & $5.4 \%$ \\
\hline No viewing & $25.7 \%$ & $0.0 \%$ & $11.4 \%$ & $25.7 \%$ & $14.3 \%$ & $14.3 \%$ & $2.9 \%$ & $5.7 \%$ \\
\hline Viewing & $54.2 \%$ & $0.0 \%$ & $12.5 \%$ & $4.2 \%$ & $0.0 \%$ & $8.3 \%$ & $8.3 \%$ & $12.5 \%$ \\
\hline $\begin{array}{l}\text { Controlled } \\
\text { viewing }\end{array}$ & $79.7 \%$ & $7.4 \%$ & $0.7 \%$ & $4.7 \%$ & $0.7 \%$ & $4.1 \%$ & $0.0 \%$ & $2.7 \%$ \\
\hline \multicolumn{9}{|l|}{ TREATMENT } \\
\hline $\begin{array}{l}\text { Paper and pencil } \\
\text { exercise }\end{array}$ & $0.0 \%$ & $0.0 \%$ & $0.0 \%$ & $0.0 \%$ & $0.0 \%$ & $\overline{100.0 \%}$ & $0.0 \%$ & $0.0 \%$ \\
\hline No viewing & $43.4 \%$ & $19.5 \%$ & $3.5 \%$ & $5.3 \%$ & $0.9 \%$ & $4.4 \%$ & $12.4 \%$ & $5.3 \%$ \\
\hline Viewing & $28.3 \%$ & $30.1 \%$ & $3.5 \%$ & $5.3 \%$ & $2.7 \%$ & $7.1 \%$ & $18.6 \%$ & $5.3 \%$ \\
\hline $\begin{array}{l}\text { Controlled } \\
\text { viewing }\end{array}$ & $42.9 \%$ & $47.6 \%$ & $1.6 \%$ & $0.0 \%$ & $4.8 \%$ & $3.2 \%$ & $0.0 \%$ & $0.0 \%$ \\
\hline Changing & $65.0 \%$ & $0.0 \%$ & $7.8 \%$ & $1.8 \%$ & $13.8 \%$ & $1.8 \%$ & $9.7 \%$ & $1.8 \%$ \\
\hline
\end{tabular}

ing animation in order to understand, how to solve the exercise. The reason that this is happening more in the treatment condition is the instant feedback that TRAKLA2 provides on each simulation, which helps students to understand when their mental models of the algorithms and data structures are not viable and they need to revise them. This example also indicates that the visiting of the engagement levels does not happen in any particular order, but can happen randomly.

(Teasley, 1997) has found that talking is correlated with better learning results. This, at least partially, explains why students learned better in the treatment condition compared to the control condition. The visualization helped them to discuss relevant topics in order to learn them. Because the topic that the participants were studying was unfamiliar to most of the participants, the conversations in the group aided students to better cope with the questions and problems that arose during the learning process. Therefore, we believe that pair-support is one of the key factors in enhancing students' learning, and it should be taken into account when designing and developing next generation learning tools and methods. Teasley has also found that transactive reasoning (Berkowitz and Gibbs, 1983) is strongly correlated with learning results, and in the future studies, we will also analyze the amount of transactive reasoning in the discussions.

In addition to the amount of discussion, we analyzed the discussion contents. Based on the analysis, we found that the students' discussions were related to the learned topic, otherwise there were no large differences. The only noticeable difference was the absence of code reading on the changing level. When we compared the distributions between conditions, there were more noticeable difference. In the treatment condition, the discussions related to the algorithm and data structure behavior and code reading were more balanced on levels no viewing, viewing and controlled viewing compared to the control condition, where students concentrated on the algorithm and data structure behavior and had very little discussion on the code. One could argue that the discussions on various levels of abstraction increased the students' understanding of the topic, and therefore, this could also be an explanation why students performed better in the post-test in the treatment condition.

\section{CONCLUSIONS}

Many studies related to the use of algorithm visualizations (AV) in teaching and learning have focused on the learning outcomes. On the one hand, (Extended) Engagement Taxonomy (EET) has been suggested to answer the question, if an AV system is effective in this respect or not. On the other hand, collaborative learning (CL) has proven to be an efficient teaching and learning method. However, very little is known about the interconnection between EET and CL.

We have investigated the use of AV in CL in many repeated studies. Our previous studies have confirmed that the engagement levels have a role to play in learning outcomes. The pairs of students that used AV 
on higher engagement levels performed better in the post-test than those pairs learning on lower engagement levels. The research in this paper has revealed that the amount of discussion in collaboration is also different between engagement levels, and increases as the engagement level increases.

Based on this study, EET not only predicts the increase in learning performance when student groups learn with visualization on higher engagement level, but also explains it by enabling students to have more discussions on topics that are relevant for learning. Thus, engagement goes hand in hand with collaboration so that the engagement taxonomy level has an influence over the collaborative learning process as well as the learning outcomes.

\subsection{Future Directions}

(Teasley, 1997) has found that transactive reasoning (Berkowitz and Gibbs, 1983) (TR) is strongly correlated with learning results. Transactive reasoning is discussion about one's own or collaboration partner's reasoning and logical thinking. TRAKLA2 exercises have interesting interconnections with the characterizations of TR categories. For example, Teasley describes prediction type TR as "explaining ..., stating a hypothesis about causal effects ... ." Moreover, the feedback request category can be characterized with a question: "Do you understand or agree with my position?"

Even though these do not correspond directly to TRAKLA2 exercises, the same elements are present in the exercise solving process. The student is supposed to predict each step in the algorithm simulation; and $\mathrm{s} / \mathrm{he}$ receives instant feedback from the exercise. Thus, this kind of framework could function as a future testbed to explain good learning results that also individual learners get in the TRAKLA2 environment or in any other environment.

\section{ACKNOWLEDGEMENTS}

This work was supported by the Academy of Finland under grant numbers 111396. Any opinions, findings, and conclusions or recommendations expressed in this material are those of the authors and do not necessarily reflect the views of the Academy of Finland.

\section{REFERENCES}

Beck, L. L. and Chizhik, A. W. (2008). An experimental study of cooperative learning in CS1. In SIGCSE' '08: Proceedings of the 39th SIGCSE technical symposium on Computer science education, pages 205-209, New York, NY, USA. ACM.

Ben-Bassat Levy, R., Ben-Ari, M., and Uronen, P. A. (2003). The Jeliot 2000 program animation system. Computers \& Education, 40(1):15-21.

Berkowitz, M. W. and Gibbs, J. C. (1983). Measuring the development of features in moral discussion. MerillPalmer Quarterly, 29:399-410.

Evans, C. and Gibbons, N. J. (2007). The interactivity effect in multimedia learning. Computers \& Education, 49(4):1147-1160.

Gall, M. D., Gall, J. P., and Borg, W. R. (2006). Educational Research: An Introduction (8th Edition). Allyn \& Bacon.

Garrison, D. R. (1993). A cognitive constructivist view of distance education: An analysis of teaching-learning assumptions. Distance Education, 14:199-211.

Grissom, S., McNally, M., and Naps, T. L. (2003). Algorithm visualization in CS education: Comparing levels of student engagement. In Proceedings of the First ACM Symposium on Software Visualization, pages 87-94. ACM Press.

Hundhausen, C. D. (2002). Integrating algorithm visualization technology into an undergraduate algorithms course: Ethnographic studies of a social constructivist approach. Computers \& Education, 39(3):237-260.

Hundhausen, C. D. (2005). Using end-user visualization environments to mediate conversations: A 'Communicative Dimensions' framework. Journal of Visual Languages and Computing, 16(3):153-185.

Hundhausen, C. D. and Brown, J. L. (2008). Designing, visualizing, and discussing algorithms within a CS 1 studio experience: An empirical study. Computers \& Education, 50(1):301-326.

Hundhausen, C. D., Douglas, S. A., and Stasko, J. T. (2002). A meta-study of algorithm visualization effectiveness. Journal of Visual Languages and Computing, 13(3):259-290.

Korhonen, A., Malmi, L., and Silvasti, P. (2003). TRAKLA2: a framework for automatically assessed visual algorithm simulation exercises. In Proceedings of Kolin Kolistelut / Koli Calling - Third Annual Baltic Conference on Computer Science Education, pages 48-56, Joensuu, Finland.

Laakso, M.-J., Myller, N., and Korhonen, A. (2009). Comparing learning performance of students using algorithm visualizations collaboratively on different engagement levels. Accepted to the Journal of Educational Technology \& Society.

Malmi, L., Karavirta, V., Korhonen, A., Nikander, J., Seppälä, O., and Silvasti, P. (2004). Visual algorithm simulation exercise system with automatic assessment: TRAKLA2. Informatics in Education, $3(2): 267-288$. 
McMahon, M. (1997). Social constructivism and the world wide web - a paradigm for learning. In Proceedings of the ASCILITE conference, Perth, Australia.

Moore, M. G. (1989). Editorial: Three types of interaction. The American Journal of Distance Education, page 16.

Myller, N., Bednarik, R., Ben-Ari, M., and Sutinen, E. (2008). Applying the Extended Engagement Taxonomy to Collaborative Software Visualization. Accepted to the ACM Transactions on Computing Education.

Myller, N., Laakso, M., and Korhonen, A. (2007). Analyzing engagement taxonomy in collaborative algorithm visualization. In Hughes, J., Peiris, D. R., and Tymann, P. T., editors, ITiCSE '07: Proceedings of the 12th Annual SIGCSE Conference on Innovation and Technology in Computer Science Education, pages 251-255, New York, NY, USA. ACM Press.

Naps, T. L. and Grissom, S. (2002). The effective use of quicksort visualizations in the classroom. Journal of Computing Sciences in Colleges, 18(1):88-96.

Naps, T. L., Rößling, G., Almstrum, V., Dann, W., Fleischer, R., Hundhausen, C., Korhonen, A., Malmi, L., McNally, M., Rodger, S., and Velázquez-Iturbide, J. Á. (2002). Exploring the role of visualization and engagement in computer science education. In Working Group Reports from ITiCSE on Innovation and Technology in Computer Science Education, pages 131-152, New York, NY, USA. ACM Press.

Palincsar, A. S. (1998). Social constructivist perspectives on teaching and learning. Annual Review of Psychology, 49:345-375.

Piaget, J. (1977). The Development of Thought: Equilibration of Cognitive Structures. Viking, New York.

Suthers, D. D. and Hundhausen, C. D. (2003). An experimental study of the effects of representational guidance on collaborative learning processes. Journal of the Learning Sciences, 12(2):183-219.

Teague, D. and Roe, P. (2008). Collaborative learning: towards a solution for novice programmers. In $A C E$ '08: Proceedings of the tenth conference on Australasian computing education, pages 147-153, Darlinghurst, Australia, Australia. Australian Computer Society, Inc.

Teasley, S. (1997). Talking about reasoning: How important is the peer in peer collaboration. In Resnick, L., Säljö, R., Pontecorvo, C., and Burge, B., editors, Discourse, Tools and Reasoning: Essays on Situated Cognition, pages 361-384. Springer, New York.

Valdivia, R. and Nussbaum, M. (2007). Face-to-face collaborative learning in computer science classes. International Journal of Engineering Education, 23:434440(7).

Vygotsky, L. S. (1978). In Cole, M., John-Steiner, V., Scribner, S., and Souberman, E., editors, Mind in Society. Harvard University Press, Cambridge, Mass. 